\title{
Direct numerical simulation of shock/turbulent boundary layer interaction in a supersonic compression ramp
}

\author{
LI XinLiang $^{1 *}$, FU DeXun ${ }^{2}$, MA YanWen ${ }^{2} \&$ LIANG Xian ${ }^{1}$ \\ ${ }^{1}$ Key Laboratory of High Temperature Gas Dynamics, Institute of Mechanics, Chinese Academy of Sciences, Beijing 100190, China; \\ ${ }^{2}$ The State Key Laboratory of Nonlinear Mechanics, Institute of Mechanics, Chinese Academy of Sciences, Beijing 100190, China
}

Received February 20, 2010; accepted March 22, 2010; published online June 21, 2010

\begin{abstract}
A direct numerical simulation of the shock/turbulent boundary layer interaction flow in a supersonic 24-degree compression ramp is conducted with the free stream Mach number 2.9. The blow-and-suction disturbance in the upstream wall boundary is used to trigger the transition. Both the mean wall pressure and the velocity profiles agree with those of the experimental data, which validates the simulation. The turbulent kinetic energy budget in the separation region is analyzed. Results show that the turbulent production term increases fast in the separation region, while the turbulent dissipation term reaches its peak in the near-wall region. The turbulent transport term contributes to the balance of the turbulent conduction and turbulent dissipation. Based on the analysis of instantaneous pressure in the downstream region of the mean shock and that in the separation bubble, the authors suggest that the low frequency oscillation of the shock is not caused by the upstream turbulent disturbance, but rather the instability of separation bubble.
\end{abstract}

compression ramp, shock/turbulent boundary layer interaction, direct numerical simulation, shock oscillation

PACS: 47.27.Eq, 47.27.Nz, 83.85.pt

\section{Introduction}

The supersonic compression ramp flow, as a typical model for studying the shock/turbulent boundary-layer interaction (STBLI), is a key flow in the scramjet inlet. The mechanism study of this flow is valuable in terms of both theories and application. During more than half a century study, large amount experimental data has been published [1]. In recent years, with the demand of hypersonic aircraft, the mechanism study of supersonic compression ramp flow becomes hot again. Besides experiments, numerical simulations also serve as a powerful tool to study the mechanism of STBLI, especially, the direct numerical simulation (DNS). The DNS without using any turbulence model will involve no modeling error. Additionally, the DNS can provide all the time-spatial messages of the complicated flow, which is

*Corresponding author (email: lixl@imech.ac.cn) very useful in the mechanism study. The geometry of a compression-ramp is simple, but the flow in a supersonic compression-ramp is rather complicated, which contains turbulence, transition, flow separation, shock waves and the interactions of these factors. The multi-factors pose great difficulties to DNS. Because the flow separation is very sensitive to numerical viscosity, the simulation requires the numerical dissipation to be very small, and the grid resolution to be high. Thus, the published DNS reports of STBLI are very few.

To the author's knowledge, Adams et al. [2,3] performed the first DNS of supersonic compression ramp flow. However, limited by the computing power, the Reynolds number of DNS cannot be as high as that of the experiment, and there is no direct comparison with the experiment result in the DNS used by Adam. Bookey et al. [4] performed an experimental study of STBLI. Different from the previous experiments, low-density gas was used as a work medium in 
Bookey et al.'s experiment, and thus the Reynolds number in Bookey et al.'s experiment is low enough to meet the standard of DNS or LES (large eddy simulation). Wu et al. [5] performed a new DNS of supersonic compression ramp flow following Bookey et al.'s experimental parameters, and the turbulence statistics agree with Bookey et al.'s experiment. To minimize the computation cost, $\mathrm{Wu}$ et al. used the recycler technique to produce the inlet fully developed boundary-layer turbulence. This method avoided the long computational domain for simulating the transition. However, the inlet turbulence provided by the recycler technique is not as reliable as that provided by transition simulation.

The oscillation of separation shock is an important feature of the shock/turbulent boundary layer interaction (STBLI). Experimental research studies report that the oscillation has two different characteristic frequencies [1,6]. The time scale of the high-frequency oscillation is $O\left(\delta / U_{\infty}\right)$, while that of the low-frequency oscillation is $O\left(10 \delta / U_{\infty}-100 \delta / U_{\infty}\right)$ [1,6], where $\delta$ is the thickness of the upwind boundary layer and $U_{\infty}$ is the free-stream velocity. The mechanism of high-frequency oscillation is deemed as the effects of boundary layer turbulent disturbance or the effects of the upstream coherent structures in the turbulent boundary layer. However, the time scale of low-frequency oscillation is much larger than that of the turbulent disturbance (or the coherent structures). Ganapathisubramani et al. [7] have found that there are coherent structure clusters (so-called "super-structures") in the turbulent boundary layer and deemed that the clusters play an important role in the low-frequency oscillation of the shock. Wu et al. [8] believe that the upwind boundary layer disturbance (including the super-structures) cannot cause the shock's low-frequency oscillation, since the correlation between the shock oscillation and the upstream disturbance is very low. They suggest that the downstream region (separation bubble) is the cause of shock's low-frequency oscillation. The reason for shock's low frequency oscillation is still unclear.

This paper has made a direct numerical simulation (DNS) of shock/turbulent boundary layer interaction (STBLI) flow in a 24-degree compression ramp with free stream Mach number 2.9. The flow parameters chosen are close to Bookey et al.'s experiment [4]. Different from Wu et al.'s simulation [5], the wall blow-and-suction disturbance in the upwind wall boundary is used to trigger the transition. Based on the analysis of instantaneous pressure in the downstream region of the mean shock and that in the separation bubble, the authors suggest that the low frequency oscillation of the shock is not caused by the upstream turbulent disturbance, but rather the instability of separation bubble. The turbulent kinetic energy budget in the separation region is also analyzed in this paper.

\section{DNS setup}

As shown in Figure 1, the computing model is supersonic flow over a 24-degree ramp, and the computational domain is also shown in this figure. The computational domain is $-335 \mathrm{~mm} \leqslant x \leqslant 51.6 \mathrm{~mm}$ streamwise ( $x=0$ denote the corner point), $L_{y}=35 \mathrm{~mm}$ in the wall-normal direction and $0 \leqslant z \leqslant 14 \mathrm{~mm}$ in the spanwise direction. The inlet of the computational domain is located at the $200 \mathrm{~mm}$ downstream leading edge of the flat-plate, and the inlet boundary condition is set by using the laminar profiles of flat-plate boundary layer flow. In the current simulation, we first simulate the two-dimensional laminar flow over a flat-plate with the leading edge, then save and use the profiles of density, velocities and temperature at the $200 \mathrm{~mm}$ downstream leading edge as the inlet boundary conditions of the three-dimensional simulation. To trigger the transition, we impose the blow-and-suction perturbation on the wall at $-305 \mathrm{~mm}$ $\leqslant x \leqslant-285 \mathrm{~mm}$ (For the detail of the perturbation, see ref. [9-11]). The amplitude of the perturbation is set as $A=0.1$. Since the perturbation is strong enough, the transition is a bypass-type transition. Table 1 shows the free-stream conditions and the condition at the location $x=-30 \mathrm{~mm}$, which is in the upstream separation bubble, where $\theta, \delta$ and $C_{\mathrm{f}}$ denote the momentum thickness, nominal thickness (99\%) and skin friction coefficient at $x=-30 \mathrm{~mm}$. The parameters of Bookey et al.'s experiment [4] and Wu et al.'s DNS [5] are also listed in this table. Table 1 shows that the parameters at $x=-30 \mathrm{~mm}$ of the current DNS differ to a small degree from those of Bookey et al.'s experiment. The reason is that the

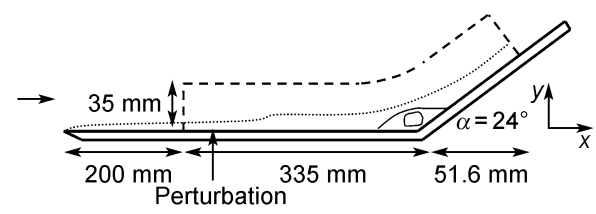

Figure 1 Schematic diagram of DNS setup.

Table 1 Flow parameters

\begin{tabular}{|c|c|c|c|c|c|c|c|c|}
\hline & \multicolumn{4}{|c|}{ Free-stream and wall } & \multicolumn{4}{|c|}{$x=-30 \mathrm{~mm}$} \\
\hline & $M a_{\infty}$ & $R e_{\infty}(\mathrm{mm})$ & $T_{\infty}(\mathrm{K})$ & $T_{\mathrm{w}}(\mathrm{K})$ & $\operatorname{Re}_{\theta}$ & $C_{\mathrm{f}}$ & $\theta(\mathrm{mm})$ & $\delta(\mathrm{mm})$ \\
\hline The current DNS & 2.9 & 5581.4 & 108.1 & 307 & 2344 & $2.57 \times 10^{-3}$ & 0.42 & 6.5 \\
\hline experiment by Bookey et al. [4] & 2.9 & 5581.4 & 108.1 & 307 & 2400 & $2.257 \times 10^{-3}$ & 0.43 & 6.7 \\
\hline DNS by Wu et al. [5] & 2.9 & 6052.6 & 107.1 & 307 & 2300 & $2.17 \times 10^{-3}$ & 0.38 & 6.4 \\
\hline
\end{tabular}


flow at $x=-30 \mathrm{~mm}$ is affected by the transition in the upstream region, and the perturbation of the current DNS is different from that of the experiment.

A high-resolution CFD software Hoam-OpenCFD developed by the authors are used to solve the Navier-Stokes equations in the curvilinear-coordinate directly:

$$
\frac{\partial\left(J^{-1} U\right)}{\partial t}+\frac{\partial\left(\hat{E}-\hat{E}_{v}\right)}{\partial \xi}+\frac{\partial\left(\hat{F}-\hat{F}_{v}\right)}{\partial \eta}+\frac{\partial\left(\hat{G}-\hat{G}_{v}\right)}{\partial \varsigma}=0
$$

where $(\xi, \eta, \varsigma)$ is the computational coordinate, and $J$ is the Jacobian coefficient of the coordinate transform form $(x, y, z)$ to $(\xi, \eta, \varsigma)$. $\hat{E}, \hat{F}, \hat{G}$ are inviscous flux and $\hat{E}_{v}, \hat{F}_{v}, \hat{G}_{v}$ are viscous flux. Steger-Warming splitting is used for the inviscous terms and then solved by using WENO-SYMBO method of Martin et al. [5,12]. WENO-SYMBO is an optimized WENO scheme in a nine-point central stencil with a fourth order accuracy, but a higher resolution than the ordinary eighth order central scheme. Since the central stencil is used, the dissipation of WENO-SYMBO is small. Furthermore, limiter-technique reported by ref. [12] is used in the WENO-SYMBO scheme. This technique closes the weighting calculation of the WENO scheme in the region where neither the velocity divergence nor the oscillation is strong [12], and it minimizes both the total dissipation and computation cost. Viscous terms are discretized by using the eighth order central scheme, and the third order TVD-type Runge-Kutta method is used for time-advance.

Mesh of the current DNS is 2160 (streamwise) $\times 140$ (wall-normal) $\times 160$ (spanwise). And the mesh is concentrated in the corner region $(-35 \mathrm{~mm} \leqslant x \leqslant 35 \mathrm{~mm})$. The mesh span in the wall unit (measured at $x=-30 \mathrm{~mm}$ ) is $\Delta x^{+}$ $\approx 4.1, \Delta y_{w}^{+} \approx 0.5$ and $\Delta z^{+} \approx 4.8$, which is much smaller than the DNS of non-separated flat-plate boundary layer. This is because the flow separation is sensitive to the numerical dissipation, and the mesh span (especially, $\Delta x$ ) must be small enough. In the current simulation, if not specified, the length is non-dimensioned by using millimeter ( $\mathrm{mm})$.

\section{Data validation}

After lengthy computation, the flow becomes statistically steady, and then the three-dimensional fields are saved and used for statistics. In the current simulation, both the spanwise average and time average are used for the statistics. Figure 2 shows the distribution of skin fraction coefficient $C_{\mathrm{f}}$. The significant growth of $C_{\mathrm{f}}$ in the region $-260 \mathrm{~mm}$ $\leqslant x \leqslant-180 \mathrm{~mm}$ denotes the occurrence of the transition. In the region $-160 \mathrm{~mm} \leqslant x \leqslant-25 \mathrm{~mm}, C_{\mathrm{f}}$ decreases smoothly, indicating that the turbulence becomes fully developed. In the downstream region, $C_{\mathrm{f}}$ goes down rapidly and shows a negative value, indicating that the separation occurs. The triangles denote the theoretical value of $C_{\mathrm{f}}$ by using Blasius turbulence formulation [9], which is identical to our DNS approximately. Figure 3 shows the mean wall pressure $\bar{p}_{w} / p_{\infty}$ as a function of $x / \delta$, where $\delta$ is the nominal boundary layer thickness at $x=-30 \mathrm{~mm}$. This figure shows that the mean pressure grows rapidly in the region $x / \delta \geqslant-3.5$, and then forms a platform region. The circles in Figure 3 denote the experimental data of Bookey et al. [4], and the error bar is set at 5\%. This figure shows that the mean wall pressure of the current DNS agrees with the experimental data, which indicates that the separation region of the current DNS agrees with the experimental data. Figures 4 and 5 show the mean velocity profile at $x=-30 \mathrm{~mm}$ and $x=-20 \mathrm{~mm}$, respectively. The two figures show that there is good agreement between the current DNS and experimental data. Figures 3-5 validate the current DNS.

\section{Flow visualization}

Figure 6 shows the two-dimensional distribution of the instantaneous temperature in the spanwise middle section $(z=7 \mathrm{~mm})$. Regions of laminar flow, transition, fully developed turbulence and separation are shown clearly in this figure. Figure 7, the locally enlarged plot of Figure 6 in the corner region, shows the clear regime of the outer boundary,

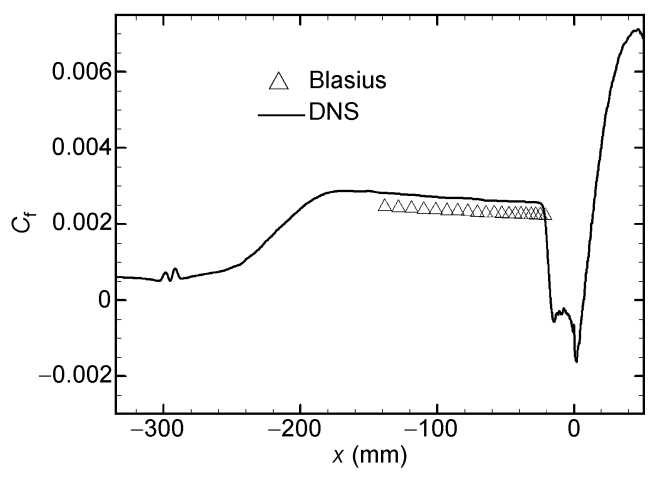

Figure 2 Distribution of the skin fraction coefficient.

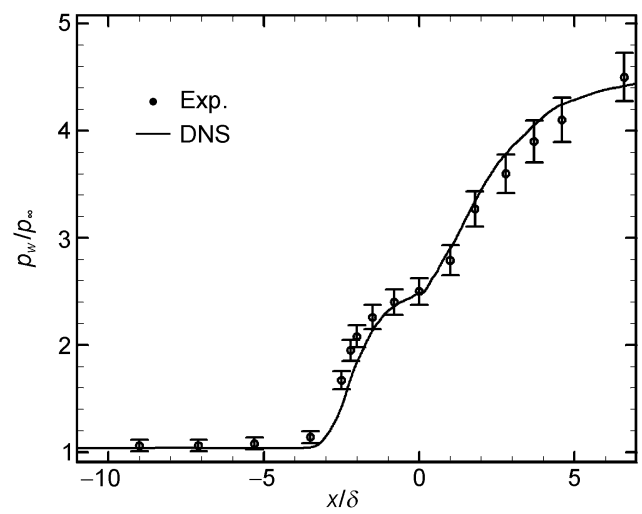

Figure 3 Distribution of the mean wall pressure. 


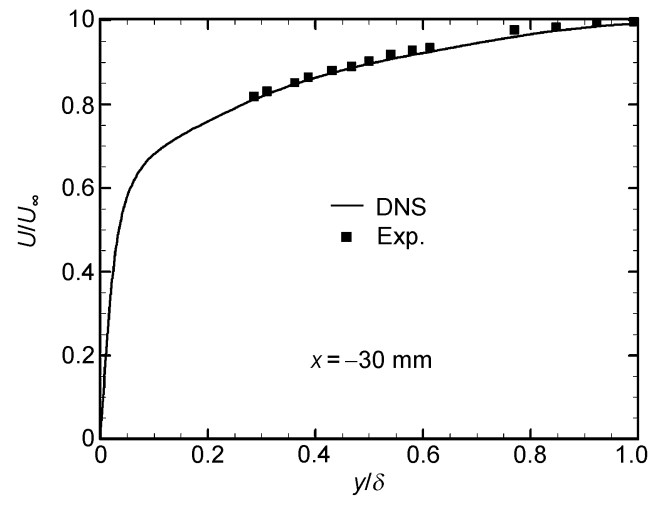

Figure 4 The mean velocity profile at $x=-30 \mathrm{~mm}$.

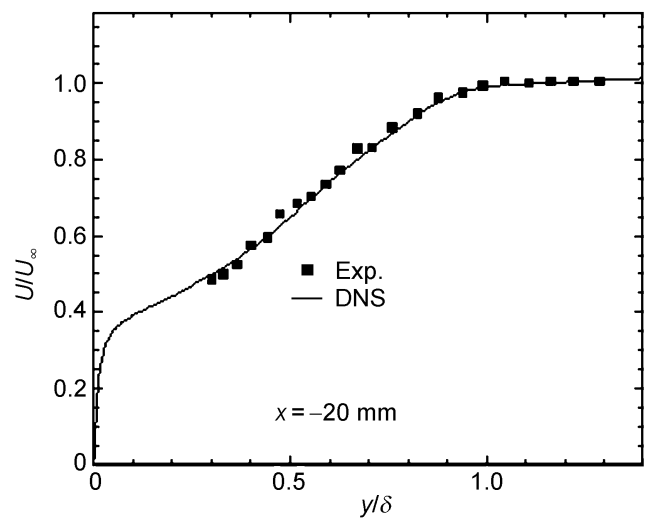

Figure 5 The mean velocity profile at $x=-20 \mathrm{~mm}$. which indicates the highly intermittent character of the outer boundary. This figure shows that the thickness of the temperature boundary layer increases rapidly in the downstream region of the separation point, and also shows that the temperature in the separation bubble is significantly higher than that in the upstream boundary layer. The shocklets in the separation bubbles are also shown in this figure.

Figure 8 shows the instantaneous numerical schlieren plots at $t=2100$ and $t=2505$ where numerical schlieren is the contour of

$$
N S=0.8 \exp \left[-10\left(\varphi-\varphi_{\max }\right) /\left(\varphi_{\max }-\varphi_{\min }\right)\right],
$$

where $\varphi=\|\nabla \rho\|$ [7]. This figure shows that the deformation of the main shock and the shocklets extends out from the boundary layer.

Figure 9 shows the instantaneous contour of streamwise velocity in a wall-parallel plane at $y_{n}=0.1 \quad\left(y_{n}^{+} \approx 4.8\right)$ and $t=2505$. Figure 10 is its locally enlarged plot. Figure 9 shows that the perturbations grow in the region $-300 \mathrm{~mm}$ $\leqslant x \leqslant-250 \mathrm{~mm}$ and then form the streamwise streaky structures. Figure 10 shows that the streaky structures are distorted in the corner region (near $x=0$ ) and then occur again in the downstream region.

\section{Turbulent kinetic energy budget}

The turbulent kinetic energy (TKE) budget equation for

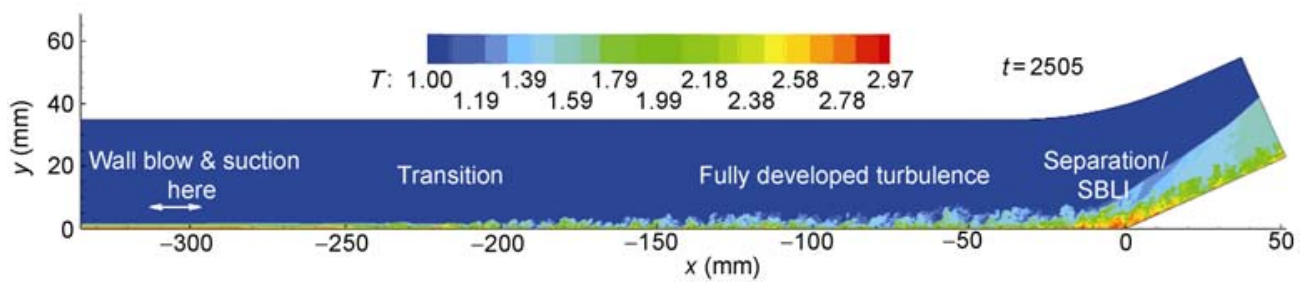

Figure 6 Distribution of the instantaneous temperature in the middle section: $z=7 \mathrm{~mm}$.

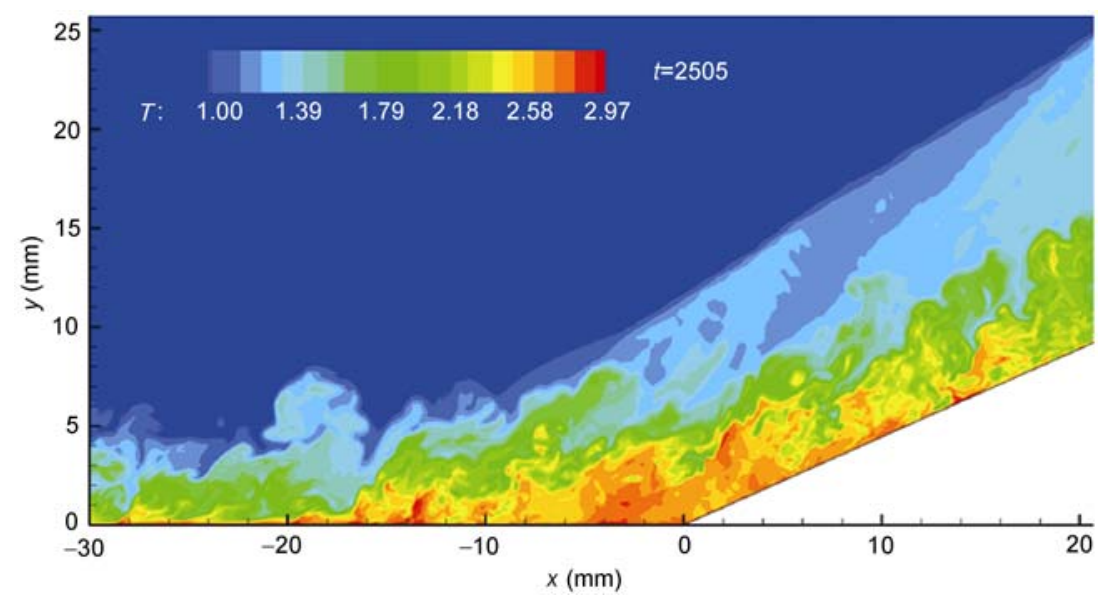

Figure 7 The locally enlarged plot of Figure 6. 

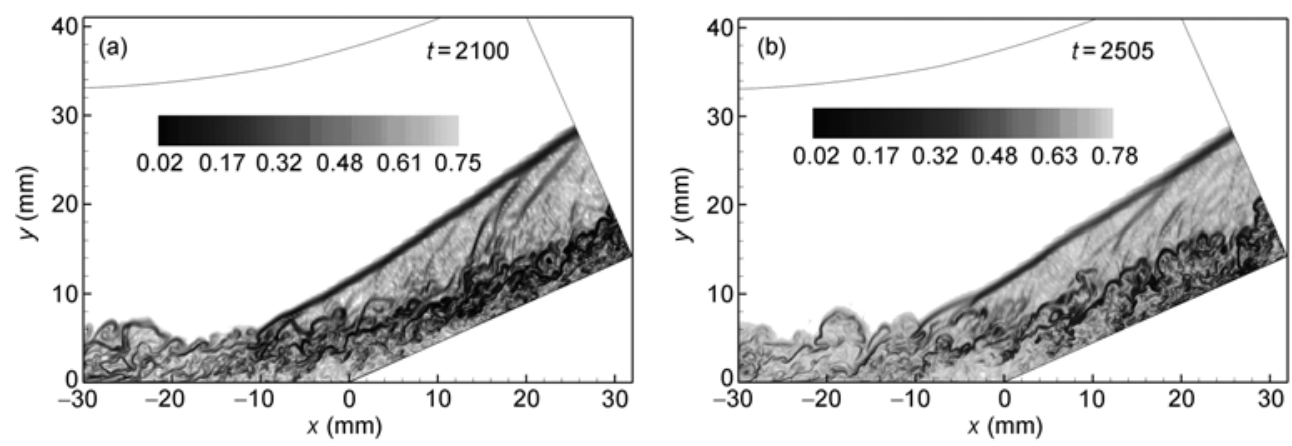

Figure 8 Instantaneous numerical schlieren plot at $t=2100$ and $t=2505$.

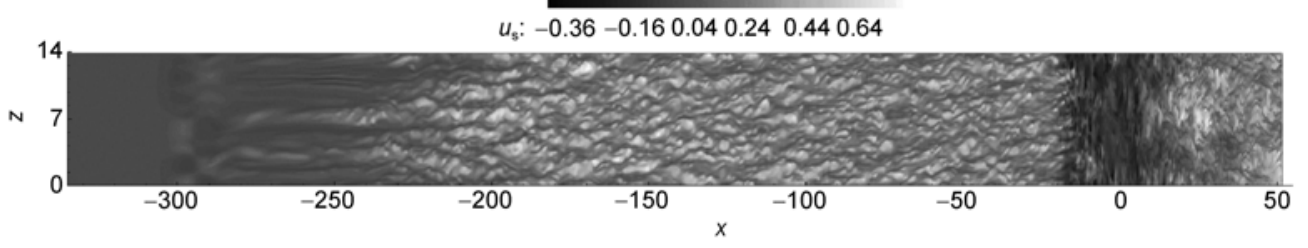

Figure 9 Contour of the instantaneous streamwise velocity at a plane parallel to the wall ( $\left.y_{n}=0.1, y_{n}^{+} \approx 4.8\right)$ at $t=2505$.

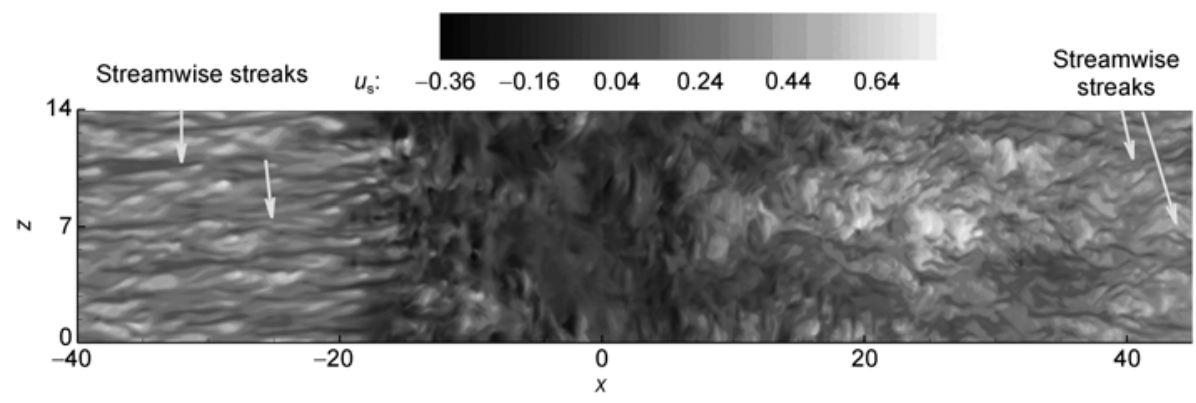

Figure 10 The locally enlarged plot of Figure 9.

compressible turbulence $[9,13]$ is:

$$
\frac{\partial}{\partial t}(\bar{\rho} K)+C=P+T+\Pi+D+M-\varepsilon,
$$

the expression of each term is shown in Table 2.

$\bar{\varphi}$ and $\tilde{\varphi}=\overline{\rho \varphi} / \bar{\rho}$ are the Reynolds average and the Favre average of $\varphi$, respectively. In the current simulation, the Reynolds average is time and spanwise average, and 142 three-dimensional fields with a time-span of 282 non-dimensional time units are used in the average. The dissipation term $\varepsilon$ can be further decomposed into the solenoidal dissipation, dilatational dissipation and the dissipation associated with the density fluctuation [9]. In many cases, the solenoidal dissipation is the most important; the dilatational dissipation and the dissipation associated with the density fluctuation are ignorable.

Figure 11 shows the two-dimensional distribution of the production term $(P)$, dissipation term $(\varepsilon)$, turbulent transport term $(T)$ and pressure-dilatation term (Pdiv) in the corner region. This figure shows that the production term is very strong in the downstream region of the separation point and in the region around the main shock. This is because the mean shear is very strong in these regions. The turbulent dissipation $\varepsilon$ is very strong both in the separation region and the downstream near-wall region. Turbulent transport term balances the production and dissipation, and the turbulent kinetic energy is transported to the near wall region and then dissipated. This figure also shows that the pressure-dilatation term (Pdiv) is significant in the region around the main shock, while this term is ignorable in other regions. This indicates that the intrinsic compressibility effect is not significant in the region which does not contain the main shock.

Figure 12 shows the one-dimensional profiles of terms in TKE equation at $x=-30 \mathrm{~mm}$, which is located in the upstream separation bubble. The profiles are very similar to those of the turbulent flat-plate boundary layer [9-11]. Tur- 
Table 2 Expression of the terms in turbulent kinetic energy (TKE) budget equation

\begin{tabular}{cccc}
\hline Expression & Meanings & Expression & Meanings \\
\hline$K=1 / 2 \overline{\rho u_{i}^{\prime \prime} u_{i}^{\prime \prime}} / \bar{\rho}$ & TKE & $C=\partial\left(\tilde{u}_{j} \bar{\rho} K\right) / \partial x_{j}$ & Convection \\
$P=-\overline{\rho u_{i}^{\prime \prime} u_{j}^{\prime \prime}} \partial \tilde{u}_{i} / \partial x_{j}$ & Production & $\varepsilon=\overline{\sigma_{i j}^{\prime} \partial u_{i}^{\prime \prime} / \partial x_{j}}$ & Dissipation \\
$T=-\partial\left(1 / 2 \overline{\rho u_{i}^{\prime \prime} u_{i}^{\prime \prime} u_{j}^{\prime \prime}}+\overline{p^{\prime} u_{j}^{\prime \prime}}\right) / \partial x_{j}$ & Turbulent transport & $D=\partial \overline{u_{i}^{\prime \prime} \sigma_{i j}^{\prime}} / \partial x_{i}$ & Viscous diffusion \\
$\Pi=\overline{p^{\prime} \partial u_{i}^{\prime \prime} / \partial x_{i}}$ & Pressure-dilation & $M=\bar{u}_{i}^{\prime \prime}\left(\partial \bar{\sigma}_{i j} / \partial x_{j}-\partial \bar{p} / \partial x_{i}\right)$ & Due to the density fluctuation \\
\hline
\end{tabular}
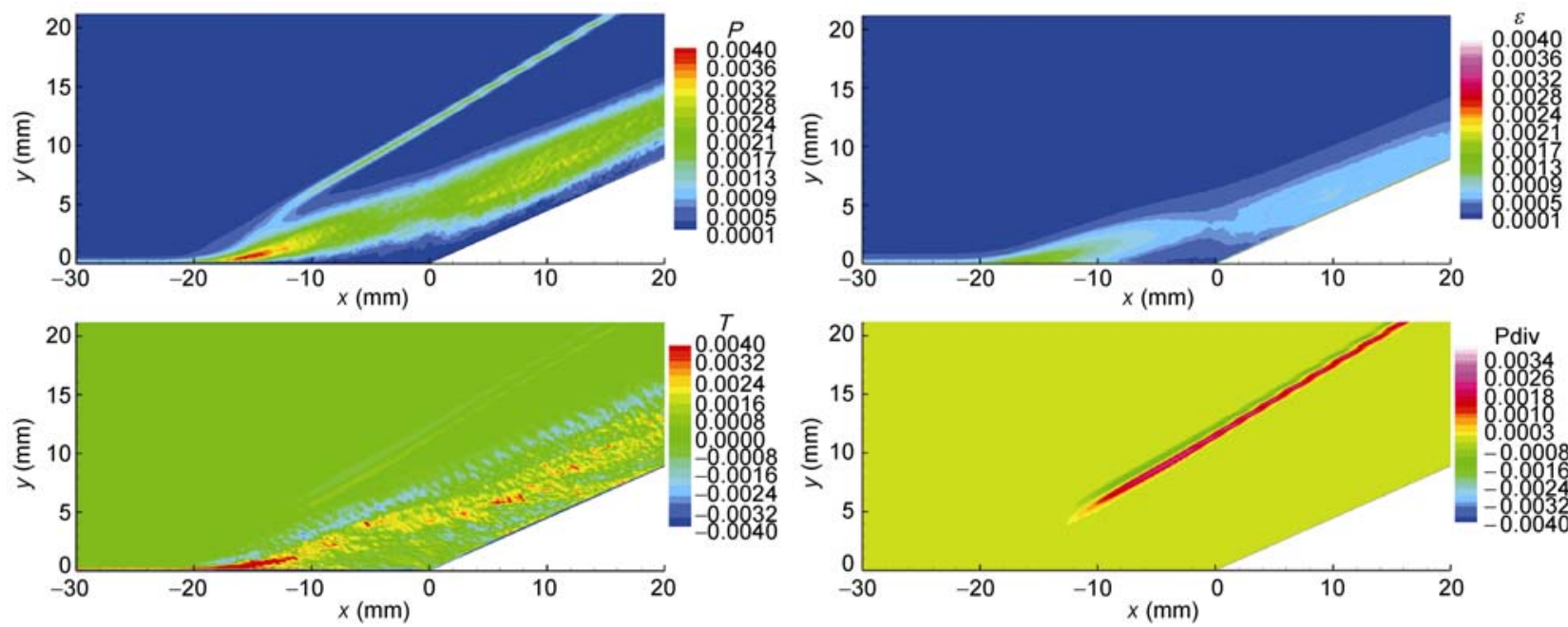

Figure 11 Distribution of the production term $(P)$, the dissipation term $(\varepsilon)$, the turbulent transport $(T)$ and the pressure-dilatation term $($ Pdiv) in the turbulent kinetic energy equation.

bulent production term reaches its peak in the transition layer, while the turbulence dissipation term reaches its peak in the wall, and the turbulent kinetic energy is transported from the far-from-wall region to the near wall region through the turbulent transport term. This figure also shows that pressure-dilation term (Pdiv), dilation dissipation term $\left(\varepsilon_{\mathrm{d}}\right)$ and the term associated with density fluctuation are ignorable. Figure 13 shows the one-dimensional profiles of the main terms in TKE equation at $x=-10 \mathrm{~mm}$ and $x=10 \mathrm{~mm}$, which are located in the separation bubble. Compared with Figure 12, the turbulent production in the separation bubble is much stronger than that in the upstream region. This fig- ure also shows that the profile of production is widely distributed in the separation bubble. Similar to that in the upstream region, the turbulent dissipation reaches its peak in the wall, and the turbulent transport term balances the turbulent production and dissipation terms.

\section{Preliminary investigation of the mechanism for shock oscillation}

The oscillation of the separation shock is an important feature of the shock/turbulent boundary layer interaction
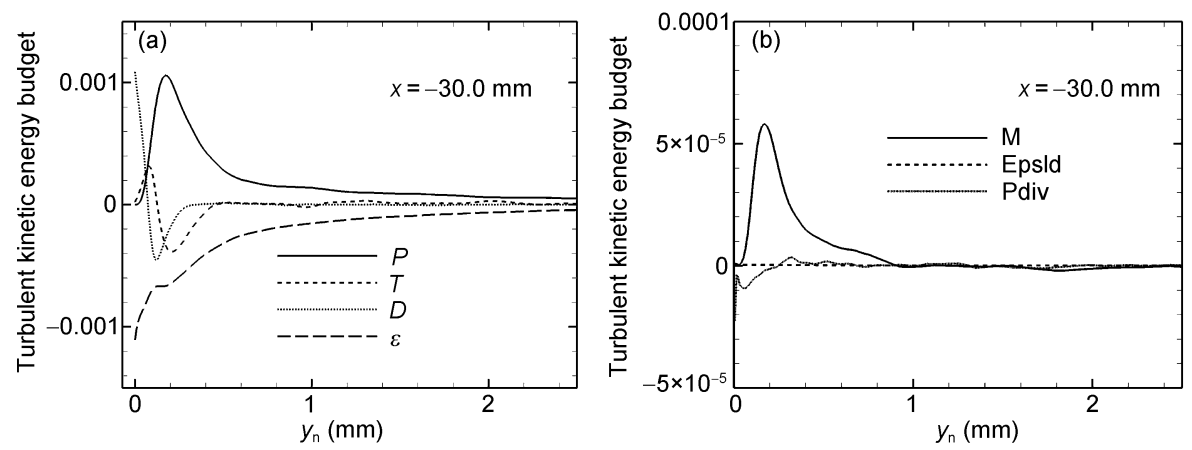

Figure 12 One-dimensional profiles of all terms in TKE equation at $x=-30 \mathrm{~mm}$. 

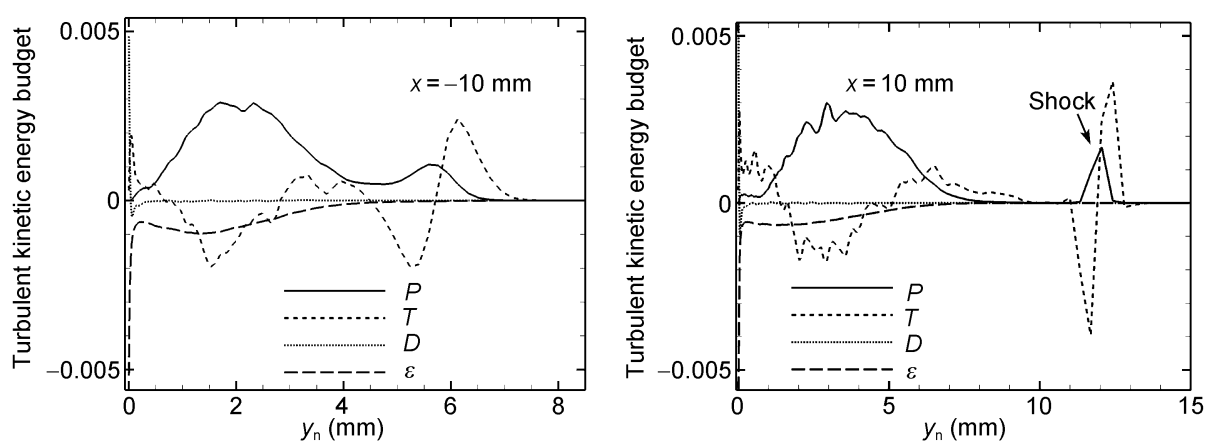

Figure 13 One-dimensional profiles of the main terms in TKE equation at $x=-10 \mathrm{~mm}$ and $x=10 \mathrm{~mm}$.

(STBLI). There are two different characteristic frequencies of the shock oscillation, and the time scale of the high-frequency oscillation is $O\left(\delta / U_{\infty}\right)$, while the time scale of the low-frequency one is $O\left(10 \delta / U_{\infty}-100 \delta / U_{\infty}\right)$. The mechanism of high-frequency oscillation is deemed as the effects of boundary layer turbulent disturbance or the effects of coherent structures in the upstream boundarylayer turbulence. However, the time scale of low-frequency oscillation is much longer than that of the turbulent disturbance (or the coherent structures). Ganapathisubramani et al. [7] have found that there are clusters of coherent structures (so-called "super-structures") in the turbulent boundary layer and deemed that the clusters of coherent structures play an important role in the low-frequency oscillation of the shock.

Figure 14 shows the visualization of coherent structures by using the iso-surface of the second invariant of velocity gradient tensor $Q$. This figure shows that coherent structures are randomly arranged, and the package of coherent structures are not clear. The authors tend to believe that the package (or cluster) of the coherent structures are not the reason for the low-frequency oscillation of the shock.

Figure 15 shows the time history of pressure disturbance $p^{\prime}=(p-\bar{p}) / p_{\infty}$ in the point $(x, y)=(-9,5.9)$, which is located in the downstream region of the shock, and the fluctuation of $p^{\prime}$ denotes the oscillation of the shock. The dash and the solid lines in Figure 15 denote the instantaneous and filtered values of $p^{\prime}$, respectively. This figure shows that low-frequency oscillation has the time scale of 100-400 non-dimensional time units, which is approximately 15-60 times of $\delta / U_{\infty}$. Figure 16 shows the pressure disturbance $p^{\prime}$ at the wall at $x=-12 \mathrm{~mm}$, which is located in the separation bubble, and it also shows the oscillation with two different frequencies. The low frequency oscillation denotes the oscillation of the separation bubble, and the time scale is close to that of the shock oscillation. So, the authors conjecture that the low-frequency oscillation of the shock is associated with the oscillation of the separation bubble, and less related to the upstream coherent structures. To further validate this speculation, we perform a laminar simulation of the compression ramp flow. The laminar simulation is two-dimensional with the same free-stream conditions as the current DNS, and the geometry is also the same as the current DNS (in the $x-y$ section). Different from the three-dimensional simulation, no perturbation is used in the two-dimensional simulation and the flow remains laminar. Figure 17 shows the instantaneous pressure in the wall at $x=-4 \mathrm{~mm}$, and this figure shows that the oscillation of wall pressure has two different time scales. The low frequency oscillation has the time scale of 400 non-dimensional time units, which is close to the time scale of low-frequency oscillation in the turbulent case. In the laminar simulation, the flow in the upstream region of the separation bubble is "clean", and there are no coherent structures or super-structures. However, the low-frequency oscillation of the separation bubble still exists. This indi-

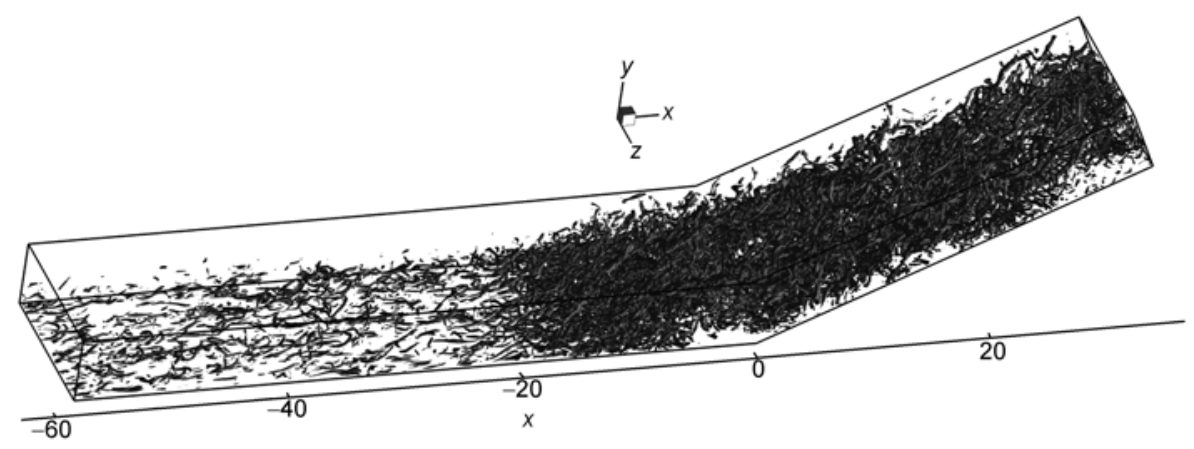

Figure 14 Visulization of the coherent structures (isosurface of the second invariant of velocity gradiant $Q$ ). 


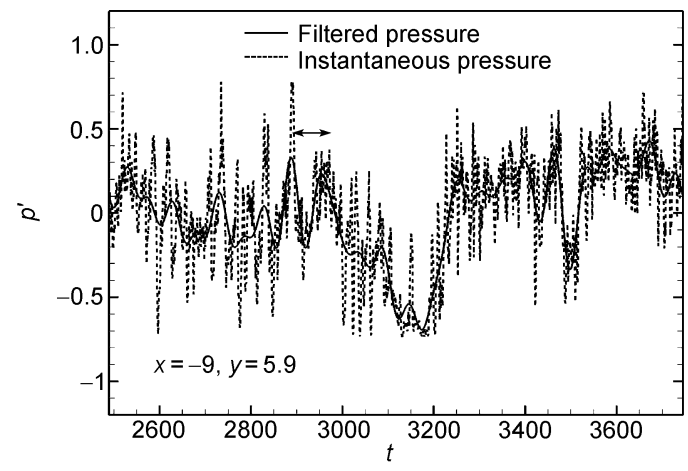

Figure 15 Time history of the pressure disturbance at the location $(x, y)=$ $(-9,5.9)$.

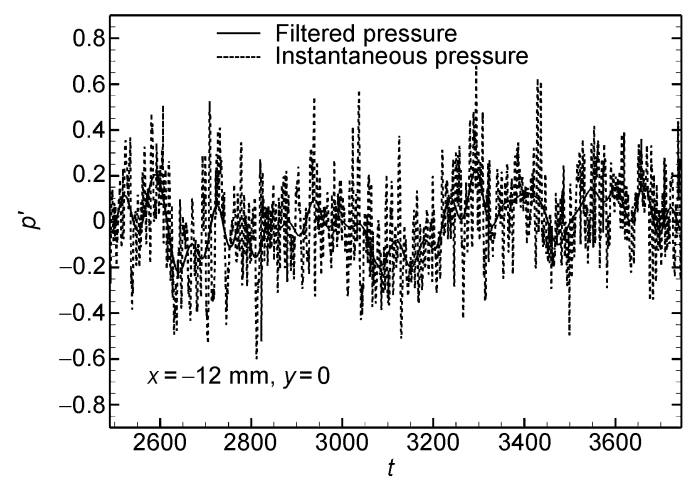

Figure 16 Time history of the pressure disturbance at the wall of $x=-12$ mm.

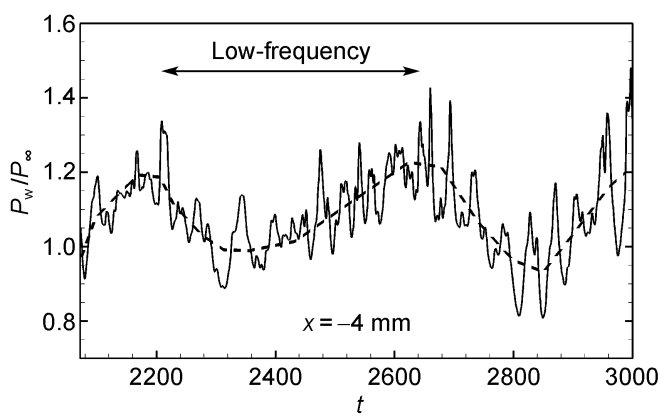

Figure 17 Time history of the pressure disturbance at the wall of $x=-4$ mm (laminar simulation).

cates that the low-frequency oscillation is not related to the upstream disturbance. So, the authors suggest that the low frequency oscillation of the shock has little relation with the upstream turbulent disturbance, and the instability of separation bubble is the suggested reason. The mechanism of low-frequency oscillation will be further investigated in the follow-up study.

\section{Conclusion}

The direct numerical simulation of shock/turbulent bound- ary layer interaction flow in a supersonic compression ramp is conducted with free stream Mach number 2.9 and 24-degree ramp-angle. The upstream wall blow-and-suction perturbation is used to trigger the transition. Both the mean wall pressure and the velocity profiles agree with those of experimental data, which validates the simulation. The turbulent kinetic energy budget in the separation region is analyzed, and the mechanism of the main shock's low-frequency oscillation is also studied.

The authors suggest that the low frequency oscillation of the shock has little relation with the upstream turbulent disturbance, which results from the instability of separation bubble.

The turbulent production term increases fast in the separation bubble, while the turbulent dissipation term reaches its peak in the near-wall region. The turbulent transport term contributes to the balance of the turbulent conduction and turbulent dissipation.

Thanks go to the Supercomputing Center of Chinese Academy of Sciences (SCCAS) and Shanghai Supercomputer Center (SSC) for providing the computing time. This work was supported by the National Natural Science Foundation of China (Grant Nos. 110632050 and 10872205), the National Basic Research Program of China (Grant No. 2009CB724100) and Projects of CAS INFO-115-BO1.

1 Dolling D S. Fifty years of of shock-wave/boundary-layer interaction research: What next? AIAA J, 2001, 39: 1517-1531

2 Adams N A. Direct numerical simulation of turbulent compression ramp flow. Theor Comput Fluid Dyn, 1998, 12: 109-129

3 Adams N A. Direct simulation of the turbulent boundary layer along a compression ramp at $M=3$ and $R e=1685$. J Fluid Mech, 2000, 420: 47-83

4 Bookey P B, Wyckham C, Smits A J, et al. New experimental data of STBLI at DNS/LES accessible reynolds numbers. AIAA Paper, 2005, AIAA-2005-309

5 Wu M, Martin M P. Direct numerical simulation of shockwave and turbulent boundary layer interaction induced by a compression ramp. AIAA J, 2007, 45: 879-889

6 Dolling D S, Or C T. Unsteadiness of the shock wave structure in attached and separated compression ramp flows. Exp Fluids, 1985, 3: 24-32

7 Ganapathisubramani B, Clemens N T, Dolling D S. Low-frequency dynamics of shock-induced separation in a compression ramp interaction. J Fluid Mech, 2009, 636: 397-425

8 Wu M W, Martin M P. Analysis of shock motion in shockwave and turbulent boundary layer interaction using direct numerical simulation data. J Fluid Mech, 2008, 594: 71-83

9 Pirozzoli S, Grasso F. Direct numerical simulation of impinging shock wave/turbulent boundary layer interaction at $M=2.25$. Phys Fluids, 2006, 18: 065113

10 Li X L, Fu D X, Ma Y W, et al. Acoustic calculation for supersonic turbulent boundary layer flow. Chin Phys Lett, 2009, 26: 094701

11 Gao H, Fu D X, Ma Y W, et al. Direct numerical simulation of supersonic boundary layer. Chin Phys Lett, 2005, 22: 1709-1712

12 Martin M P, Taylor E M, Weirs V G. A bandwidth-optimized WENO scheme for the effective direct numerical simulation of compressible turbulence. J Comput Phys, 2006, 220: 270-289

13 Li X L, Fu D X, Ma Y W. DNS of compressible turbulent boundary layer around a sharp cone. Sci China Ser G-Phys Mech Astron, 2008, 51: 699-714 